\title{
High-quality AIN films grown on chemical vapor-deposited graphene films
}

\author{
Bin-Hao Chen ${ }^{1}$, Hsiu-Hao Hsu ${ }^{2}$ and David T.W. Lin ${ }^{2}$ \\ ${ }^{1}$ Department of Materials and Energy Engineering, Far East University, No.49, Chung Hua Rd., Hsin-Shih. Tainan County 744, Taiwan \\ (R.O.C.) \\ Institute of Mechatronic System Engineering, National University of Tainan, No. 33, Sec. 2, Shu-lin St., Tainan City 700, Taiwan (R.O.C.)
}

\begin{abstract}
We report the growth of high-quality AlN films on graphene. The graphene films were synthesized by CVD and then transferred onto silicon substrates. Epitaxial aluminum nitride films were deposited by DC magnetron sputtering on both graphene as an intermediate layer and silicon as a substrate. The structural characteristics of the AlN films and graphene were investigated. Highly c-axis-oriented AlN crystal structures are investigated based on the XRDpatterns observations.
\end{abstract}

\section{Introduction}

The Graphene is single atom thick two-dimensional material, which has the structure of hexagonal carbon atoms that are $\mathrm{sp} 2$ bonded, and have high mobility and optical transparency, flexibility, robustness and environmental stability. These characteristics mean that the broader application of graphene has attracted great interest [1-7]. Perfect graphene is a two-dimensional crystal composed of carbon atoms, as shown in Figure 1, in which the atoms are arranged in a honeycomb hexagon, and if they form in pentagons or heptagons then these constitute defects. Current methods of growing graphene are divided into two types, graphene derived from graphite, and that synthesized from other sources. The graphite approach is based on mechanical exfoliation, in which the atoms are chemically cleaved from each other. Graphene synthesis can be carried out using a silicon carbide epitaxial growth method or a chemical vapor deposition method. However, mechanically exfoliating graphene from bulk graphite is not suitable for the synthesis of large surface area devices, nor for use in practical device fabrication processes. In contrast, chemical vapor deposition (CVD) is can be used with large surface area applications and has a relatively low cost [4,7-10]. The low pressure CVD (LP-CVD) method of growing graphene on a $\mathrm{Cu}$ substrate has been widely reported in the literature, there are many studies on the mechanism of the resulting materials [3,11,12]. In particular, it has been shown that a large surface area of monolayer graphene with excellent quality can be synthesized by LP-CVD.

Our experiments used graphene as an intermediate layer in epitaxial aluminum nitride films, with aluminum nitride (AIN) being a III-V compound that has a wurtzite hexagonal close-packed structure. Aluminum nitride has a variety of excellent physical properties, such as a high melting point $(3273 \mathrm{~K})$, high thermal conductivity (285 $\mathrm{W} / \mathrm{mK})$, high direct band gap (6.2 Ev), good dielectric constant (8.5) and high hardness (about $~ 2 \times 103 \mathrm{kgf} \mathrm{mm}$ 2) $[13,14,15]$. Common ways of depositing aluminum nitride films include DC and RF magnetron sputtering [13-18], chemical vapor deposition [19, 20], and molecular beam epitaxy [21]. However, the CVD and MBE methods can only be used at high temperatures, and this limits the base materials that can be used, and if the resulting grains are too big then this will lead to a rough surface, and so it will not be possible to easily manufacture electrodes on the films. In contrast, reactive RF sputtering offers good growth patterns, with the advantages of low temperature deposition, surface smoothness, fast deposition rate, and low complexity.

The use of graphene thin film epitaxial growth of III-v nitride the advantage that graphene has atoms arranged similar to a hexagonal wurtzite structure. In addition, the low migration barrier of III-V metals on graphene allows adatoms to diffuse readily on the surface at reduced growth temperatures. The use of pristine graphene can thus enable large adatom diffusion lengths during nucleation, which may promote the formation of large two-dimensional islands [22]. The graphene films were synthesized in this paper by CVD, and then transferred onto silicon substrates. Epitaxial aluminum nitride films were deposited by DC magnetron sputtering on both graphene as an intermediate layer and a silicon substrate. The structural characteristics of the AlN films and graphene were investigated and analyzed, and the results of this a reported below. 


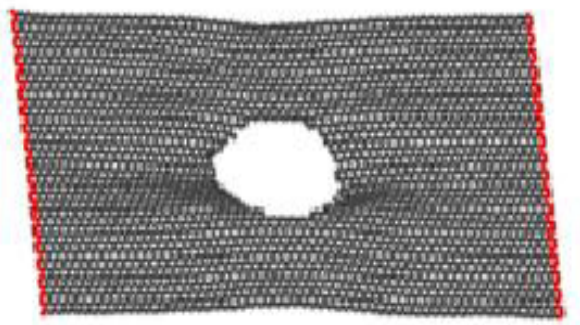

Figure 1. Perfect graphene is a two-dimensional crystal composed of carbon atoms.

\section{Experiments}

\subsection{Graphene synthesis}

Synthesis flow chart graphene has three sections, as shown in Figure2.In our experiments the copper substrate was first pre-treated, and then cut into piece of the desired size. Hydrochloric acid (HCL) was used to clean the surface to remove oxide impurities, and then DI water and ethanol were used to remove the HCL liquid residues, as shown in Figure 3.

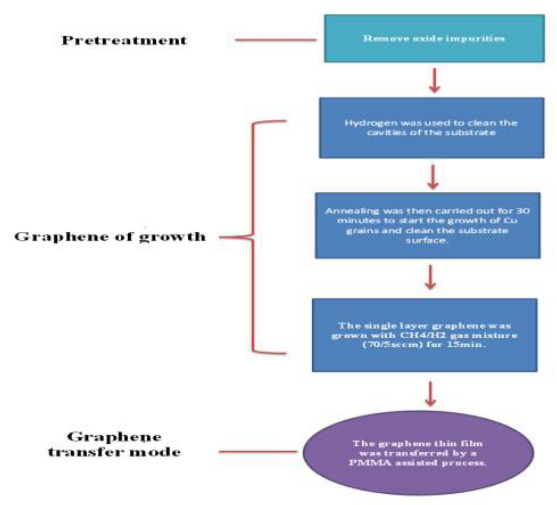

Figure 2.Synthesis of Graphene flow chart shown

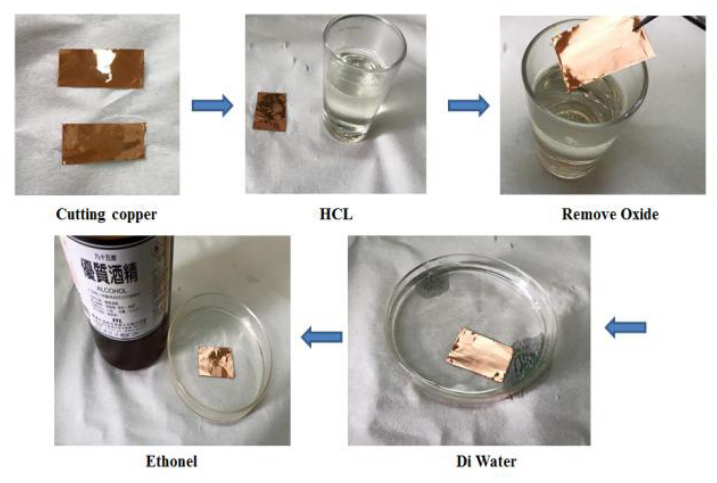

Figure 3.Graphene pre-treated process
Before graphene synthesis a $10 \mathrm{sccm}$ flow of hydrogen was used to clean the cavities of the substrate, Annealing was then carried out for 30 minutes to start the growth of $\mathrm{Cu}$ grains and clean the substrate surface. Graphene was then deposited at $1050{ }^{\circ} \mathrm{C}$ for 15 minutes using a precursor mixture of $\mathrm{CH} 4(5 \mathrm{sccm})$ and $\mathrm{H} 2(70 \mathrm{sccm})$, as shown in Figure4. Finally, the graphene thin film was transferred by a PMMA assisted process in which copper was etched by $5 \%$ ferric nitrate, and the graphene thin film was transferred onto a $\mathrm{Si}$ wafer. The PMMA was then dissolved in acetone, as shown in Figure 5 and 6.

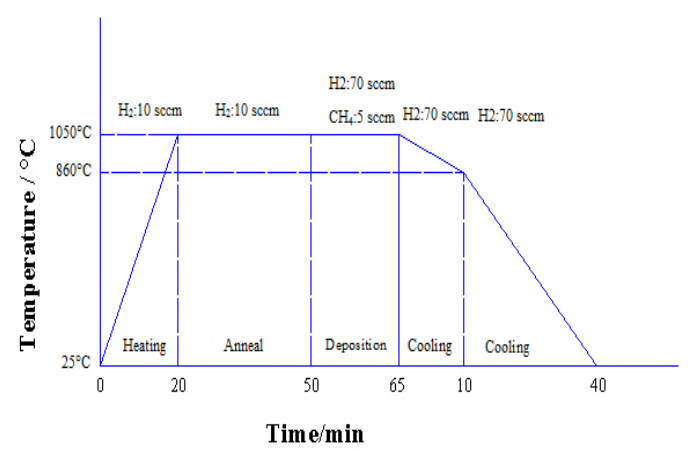

Figure 4.Heating curve diagram of graphene.

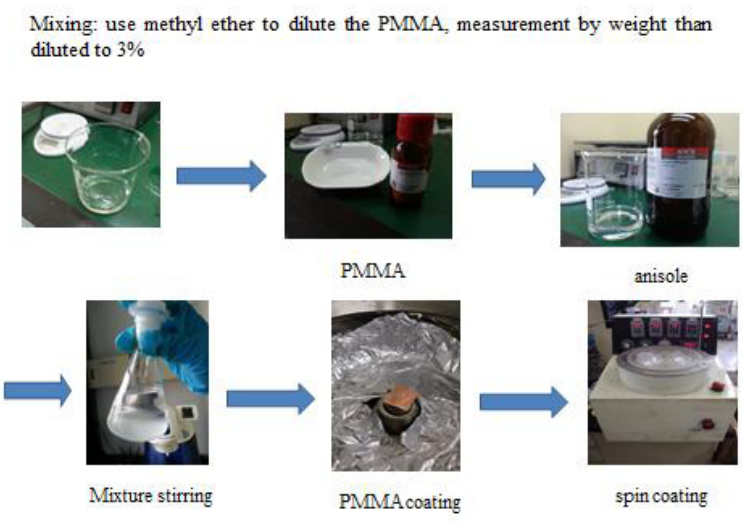

Figure 5.PMMA (Poly(methyl methacrylate) ) deployment and coat of sample

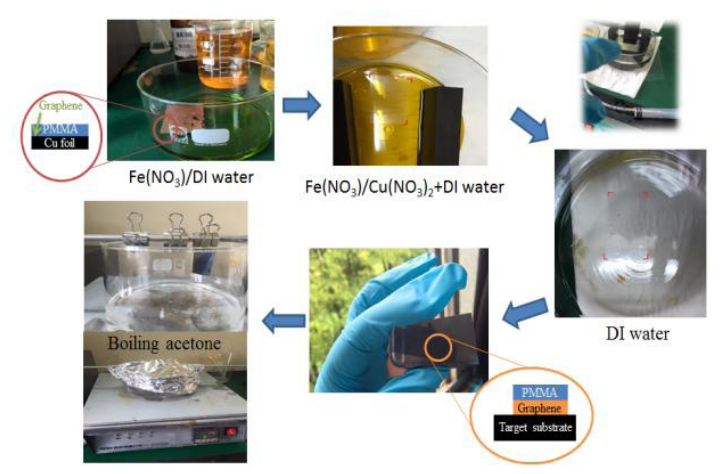

Figure 6.Graphene transfer mode 


\subsection{Aluminum nitride thin film process}

After confirming the presence of graphene on silicon, the epitaxial aluminum nitride films were deposited by DC magnetron sputtering. Aluminum nitride thin films were grown using a high purity 4N 2 inch aluminum target, and high purity argon and nitrogen were used as the reaction gas, with a nitrogen concentration of $40 \%$, temperature of $400{ }^{\circ} \mathrm{C}$, pressure of $8 \mathrm{mT}$ Torr and power $250 \mathrm{~W}$ at 3 hours. The resulting AlN film was then analyzed by XRD.

\section{Results and discussion}

Figure 7 shows an optical microscopy (OM) image of the graphene film on the silicon substrate. Since graphene is only one atom thick it is difficult for the naked eye to observe. However, the different colors and intensities of the light reflected from the substrate and graphene can be used to distinguish layers, and silicon substrates with oxide layers of different thicknesses also have different colors.

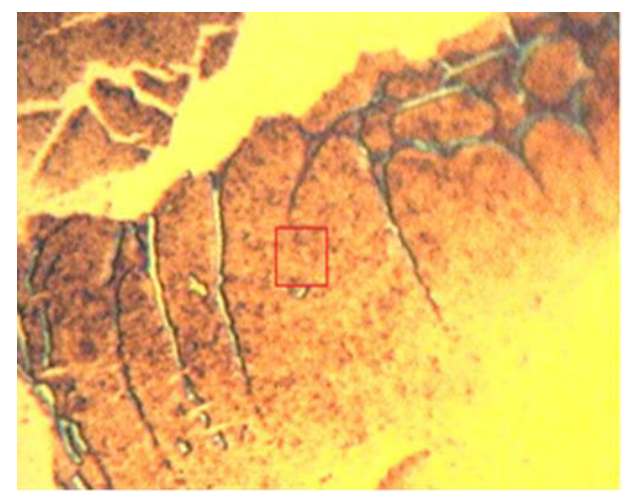

Figure 7. Optical microscope image shows a CVD-graphene film grown on $\mathrm{Cu}$-foil after transfer to a $\mathrm{Si}$ wafer.

Scanning electron microscopy (SEM) was used to observe the surface of the thin film, as shown in Figure8, in which the boundary of the copper lattice can be clearly seen. Since the number of graphene layers determines the brightness of different regions, when there is low electron absorption then brighter areas will be seen. The dark areas may be due to of several factors, such as the carbon content or substrate structure.

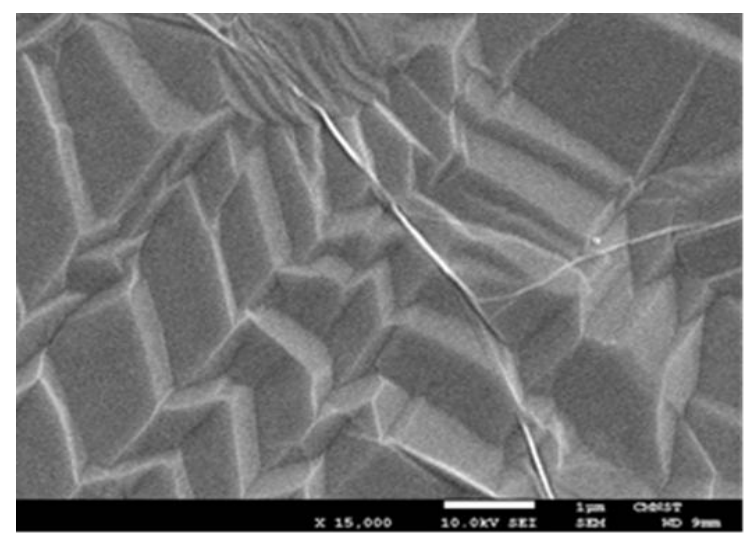

Figure 8.SEM image of a graphene on $\mathrm{Cu}$ substrate.
Figure 9 shows there are three peaks, the D peak, G peak and 2D peak at $2648 \mathrm{~cm}-1,1344 \mathrm{~cm}-1$, and $1585 \mathrm{~cm}-$ 1 , respectively, due to the different single layer or multilayer graphene structures, with the intensity of the Ramen spectra varying as the number of layers increases. Based on these characteristics, we can determine the number of layers of graphene. D peak is disordered vibration peak graphene, graphene because structural defects, away from the center of the Brillouin zone caused by the movement of the lattice, the defect when the sample is large, the higher its D peak. G Peak, on behalf of graphene good or bad, by stretching vibration between carbon atoms SP2 caused, in the center of the Brillouin zone E2g phonon vibrations. 2D peak double resonance Raman phonon peak use its judgment of graphene layers. Due to the different single or multigraphene structure, with increasing number of layers, shape, and intensity of seats in the Raman spectrum has not different. The Raman intensity ratio of ID/IG can be used to assess the quality of graphene, and in general the ID/IG value for high quality graphene will not exceed $10 \%$. The results thus show that our graphene is high quality, as it has an ID/IG ratio of about 0.13 . A previous study [7] on the synthesis of graphene by CVD reported that when I2D/IG (2.4) > 2 this indicates monolayer graphene, while $1<\mathrm{I} 2 \mathrm{D} / \mathrm{IG}(1.1)<2$ indicates bilayer graphene, and I2D/IG $(0.4)<1$ is a signature of trilayer graphene. Figure 5 shows the I2D/IG ratio of 1.96 , and this, along with the Raman spectra, also shows the existence of graphene on a Si substrate.

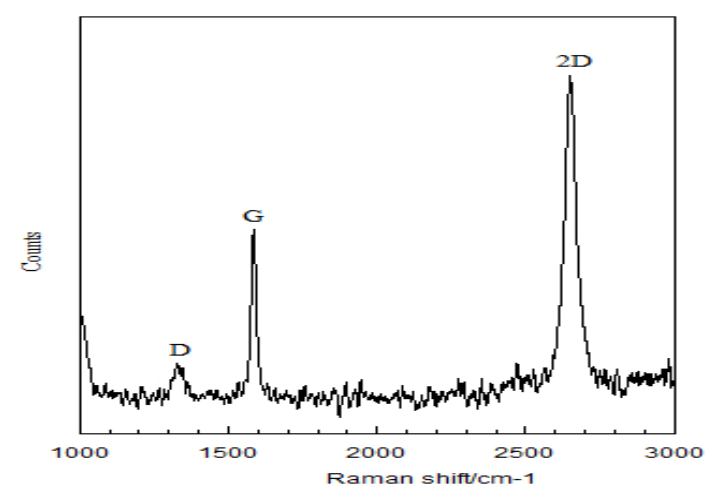

Figure 9.Raman spectra of graphene on Si substrate.

The XRD pattern of as-prepared sample is shown in Figure 10 andFigure 11. It can be clearly seen that all the peaks can be indexed by hexagonal wurtzite structure AlN. The (002) peak is the highest one in all of the peaks, which suggests that the $\mathrm{C}$-axis growth direction. The measured XRD patterns show prominent diffraction peaks corresponding to the $\left(\begin{array}{lll}1 & 0 & 0\end{array}\right),\left(\begin{array}{lll}0 & 0 & 2\end{array}\right)$ and $\left(\begin{array}{lll}1 & 0 & 1\end{array}\right)$ crystal planes of the hexagonal wurtzite phase of AlN, respectively. These crystal planes value can see the peak value of the graphene substrate intensity all to be higher than the Silicon substrate. Thus can be seen, graphene can let adatoms diffuse easily on the surface, so conducive to the epitaxial growth of aluminum nitride. 


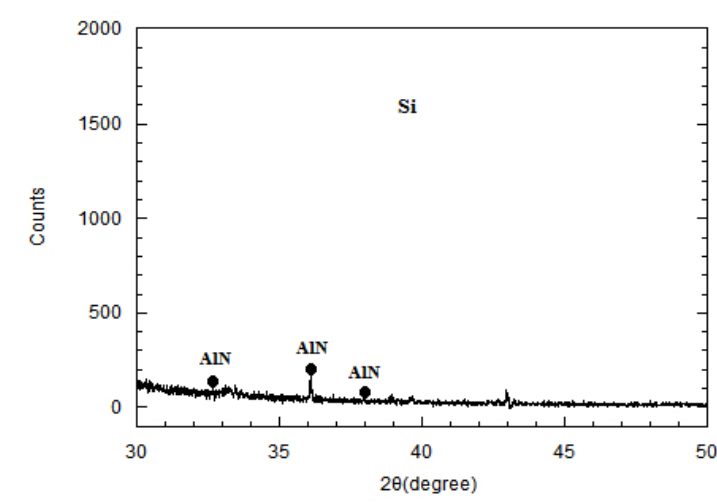

Figure 10.XRD of ALN on Si substrate.

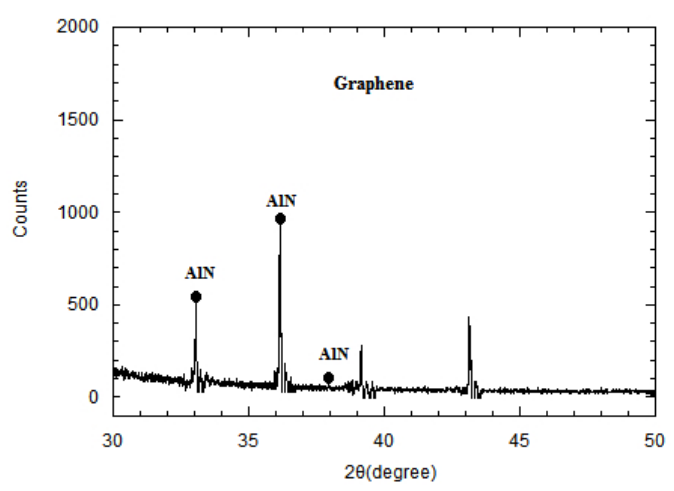

Figure 11.XRD of ALN on grapheme substrate.

\section{Conclusion}

The ID/IG ratio of about 0.13 indicates that the graphene produced in this work is high quality, while the $\mathrm{I} 2 \mathrm{D} / \mathrm{IG}$ ratio of 1.96) and the Raman spectra results also prove the existence of graphene on the Si substrate in this study. Epitaxial aluminum nitride films were deposited by DC magnetron sputtering on both graphene as an intermediate layer and a silicon substrate. The structural characteristics of the AlN films and graphene were investigated. The XRD patterns of the as-prepared samples show that the peak values of the graphene substrate are all to be higher than those of the silicon substrate. This shows that graphene can let the adatoms diffuse easily on the surface of the substrate, and this is conducive to the epitaxial growth of aluminum nitride.

\section{Acknowledgements}

The authors are thankful for the financial support of this research from the National Science Council of Taiwan through the grant no. MOST103-2221-E-269-010.The authors also express their gratitude to the Energy R\&D Foundation of the Energy Bureau of the Ministry of Economic Affairs, Taiwan, for providing financial support.

\section{References}

1. Y.H. Zhang, Z.Y. Chen, B. Wang, Y.W. Wu, Z. Jin, X.Y. Liu, G.H. Yu,Materials Letters 96,149(2013)

2. I. Vlassiouk , P. Fulvio, H. Meyer, N. Lavrik, S. Dai, P. Datskos, S. Smirnov, Carbon 54,58(2013)

3. X. Li, W. Cai, J. An, S. Kim, J. Nah, D. Yang,R. Piner, A. Velamakanni,I. Jung, E. Tutuc,S.K.Banerjee, L. Colombo, R.S. Ruoff, Science 324,1312(2009)

4. P. Trinsoutrot, C. Rabot, H. Vergnes,A. Delamoreanu,A. Zenasni, B. Caussat,Surface \& Coatings Technology 230,87(2013)

5. Y. Zhang,Y.W. Tan, H.L. Stormer, P. Kim,Nature438,201(2005)

6. Y.M. Lin, C. Dimitrakopoulos, K. A. Jenkins,D. B. Farmer, H.Y. Chiu,A. Grill, Ph. Avouris, Science 327,662(2010)

7. Z. Tu, Z. Liu, Y. Li , F. Yang, L. Zhang, Z. Zhao, C. Xu , S. Wu , H. Liu , H. Yang, P. Richard,Carbon 73,252(2014)

8. A.K. Geim, P. Kim,Scientific American298,90 (2008)

9. Y. Zhang, L. Zhang,C. Zhou, Accounts of Chemical Research46,2329(2013)

10. G. Deokar, J. Avila, I.Razado-Colambo , J.L. Codron, C. Boyaval, E. Galopin,M.C. Asensio , D. Vignaud,Carbon 89,82(2015)

11.P.Y.Huang, C.S.Ruiz-Vargas, A.M.van der Zande, W.S.Whitney, M.P.Levendorf, J.W.Kevek, S.Garg, J.S.Alden, C.J.Hustedt, Y.Zhu,J. Park,P. L. McEuen, D.A.Muller, Nature 469,389(2011)

12. X. Li, C.W. Magnuson, A. Venugopal, R.M. Tromp, J.B. Hannon, E. M. Vogel,L. Colombo, R.S. Ruoff, J. Am. Chem. Soc.133,2816(2011)

13. H.Y. Liu, G.S. Tang, F. Zeng , F. Pan,Journal of Crystal Growth 363,80(2013).

14. V.I. Dimitrova, D.I. Manova, D.A. Dechev, Vacuum49,193(1998)

15. K.A Aissa, A. Achour,J. Camus, L.L. Brizoual, P.Y. Jouan, M.A.Djouadi,Thin Solid Films550,264(2014)

16. Z. Vashaei, T. Aikawa, M. Ohtsuka, H. Kobatake, H. Fukuyama, S. Ikeda, K. Takada, Journal of Crystal Growth311,459(2009)

17. M.A. Moreira, I. Doi, J.F. Souza, J.A. Diniz, Microelectronic Engineering88,802(2011)

18. I.Safi, , Surface and Coatings Technology 127,203(2000)

19. R. Boichot, A. Claudel, N. Baccar, A. Milet, E. Blanquet, M. Pons,Surface \& Coatings Technology 205,1294(2010)

20. L. Guo, G. Chen, Y. Zhu, X. Duan, H. Ye, Journal of Crystal Growth 426,49(2015)

21. B. Liu, J. Gao, K.M. Wu, C. Liu,Solid State Communications 149,715(2009)

22.Z.Y. Al Balushi,T. Miyagi, Y.C. Lin , K. Wangb, L. Calderin,G. Bhimanapati, J.M.Redwinga, J.A. Robinson, Surface Science 634,81(2015) 